Revista de Psicología Vol. 30 (2), 2012 (ISSN 0254-9247)

\title{
Estabilidad y variabilidad en la adquisición de seis hitos motores durante la infancia temprana
}

\author{
Ernesto Pollitt, Ph.D ${ }^{1}$ \\ Universidad de California en Davis, EEUU
}

El propósito del trabajo es mostrar, mediante evidencia proveniente de estudios científicos con infantes sanos y bien nutridos y con poblaciones infantiles con altas tasas de desnutrición e infecciones, que el orden en que se presenta la emergencia de los hitos motores gruesos es estable en estos dos grupos. Por otro lado, la variabilidad en la edad de adquisición de los mismos entre los infantes en riesgo es mayor que en los nińos sanos y bien nutridos. Los resultados sugieren que factores genéticos pueden contribuir a la estabilidad en el orden de emergencia y que factores socioculturales y de salud pueden haber contribuido al origen de las diferencias en la edad de adquisición. La información tiene importancia teórica y aplicada para la evaluación del desarrollo motor con el propósito objetivo de identificar los determinantes de dicha área del desarrollo.

Palabras clave: desarrollo motor, infancia temprana

Stability and variability in the acquisition of six motor mile-stones during early infancy The purpose of this work is to show, through evidence derived from scientific studies of healthy and well-nourished infants as well as infants selected from populations with high rates of chronic infection and malnutrition, that the order in the emergence of motor milestones is stable among these two groups. On the other hand, among the children at risk, the variance in the age of emergence of the same milestones was wider than that among the healthy and well-nourished children. The similarities between groups suggest that genetic factors explain the stability of order of emergence of the milestones, while social and cultural factors may explain the differences between groups in the variance in the age of acquisition. The information has theoretical and applied value in the evaluation of motor development in order to identify the determinants of this area of development.

Keywords: motor development, early infancy

1 Profesor Emérito de la Universidad de California en Davis, Profesor Honorario de la PUCP y Miembro de la Academia Nacional de Ciencias del Perú. Contacto: Av. Aurelio Miró Quesada 610, Lima 27, Perú; epollitt@ucdavis.edu 

Tradicionalmente, los estudios sobre desarrollo motor han pasado de ser principalmente descriptivos, que respondían a la pregunta ¿Cómo crecen los infantes?, a los prescriptivos, dirigidos a responder ¿Cómo deberían crecer?, asumiéndose la presencia de etapas universales en el desarrollo motor. Esta noción implica que los niños no pueden saltarse una etapa y que los hitos motores no pueden ocurrir simultáneamente $o$ en un orden diferente.

Con el objetivo primario de obtener un estándar internacional con el cual se pueda comparar las diferentes poblaciones en el mundo, se llevó a cabo por la Organización Mundial de la Salud, el estudio llamado Normativo del Desarrollo Motor (OMSDM) (De Onis, Garza, Onyango, \& Martorell, 2006; Wijnhoven et al., 2004). El estudio permitiría llegar a juicios de valor importantes, debido a que el crecimiento físico normal es considerado un indicador de la salud infantil.

El OMSDM se concentró en el estudio de 6 hitos motores gruesos distintivos: sentarse sin apoyo, caminar a gatas (gatear), pararse con apoyo, caminar con apoyo, pararse solo y caminar solo, que fueron considerados fundamentales para la adquisición de la locomoción bípeda (WHO Multicentre Growth Reference Study Group, 2006a). Los investigadores no asumieron que los hitos motores seguían una secuencia fija. El estudio incluyó cinco países (Ghana, India, Noruega, Omán y los Estados Unidos), que constituyeron, en su totalidad, una muestra representativa de las regiones más grandes en el mundo. El OMSDM es el primer estudio longitudinal con un protocolo de trabajo estandarizado hecho con el propósito de describir el desarrollo motor grueso durante los dos primeros años de vida en infantes sin problemas de salud, ni privaciones ambientales y económicas y que hayan nacido en ciudades ubicadas a una altitud por debajo de los 1500 metros sobre el nivel del mar que viven en diferentes países del mundo (De Onis, Garza, Victora, Onyango, Frongillo \& Martines, 2004). 
La muestra restringió la variabilidad social y económica entre los participantes y, simultáneamente, mantuvo la variabilidad étnica y genética internacional. Los datos sobre los hitos motores adquiridos por cada infante provenían de las madres (o cuidadoras) de los niños participantes y de la observación llevada a cabo por los asistentes de investigación. En la evaluación directa, hecha por las madres o cuidadoras de los infantes, se registraban la fecha en que un hito se observó por primera vez. Por otro lado, las asistentes de investigación registraban la fecha cuando se demostraba la presencia del hito durante una visita al hogar del infante.

Si bien los datos del estudio normativo OMSDM fueron usados para generar trayectorias de logros provenientes de 816 infantes, éstos solo serán de utilidad en la medida que se basen en datos derivados de muestras grandes, heterogéneas y cultural y económicamente diversas (Feldman, 2007). Sin embargo, diversos estudios que buscan normas para el desarrollo motor, están realizados en grupos de infantes sanos provenientes de nivel socioeconómico medio y alto. Diversos estudios transculturales, indican que la noción de una secuencia universal es un concepto erróneo dentro del desarrollo motor (Adolph, 2010).

Basados en evidencia proveniente de estudios científicos con infantes sanos y bien nutridos y con poblaciones infantiles con altas tasas de desnutrición y enfermedades infecciosas, el objetivo de este trabajo es demostrar que la emergencia de los hitos motores es estable, mientras que existe variabilidad en la edad de adquisición de los mismos. La información tiene importancia teórica y aplicada para entender las dificultades para la formulación de estudios normativos del desarrollo motor.

En el estudio normativo OMSDM aproximadamente el 90\% de los participantes siguieron una secuencia fija en el caso de cinco hitos (sentarse sin apoyo, pararse con apoyo, caminar con apoyo, sentarse sin apoyo y caminar solo) (Fig. 1). La excepción fue el gatear. En este caso hubo tres patrones diferentes incluyendo al 86\% de los casos. El 41.7\% de los participantes gateaban antes de poder pararse con apoyo y el $36.1 \%$ gateaban antes de poder caminar con apoyo (WHO Multicentre Growth Reference Study Group, 2006b). Otros estudios indican que 
niños africanos, no gatean, y los niños que lo hacen, lo realizan a edades más tardías (Kilbride, 1980). Así mismo, el 17\% de los niños británicos omiten el gateo ( $10 \%$ se queda un mayor tiempo en la posición de sentado, y el 7\%, simplemente se puso de pie y caminó) (Robson, 1984). Este hallazgo es importante, pues el gateo es una habilidad que figura en las escalas de Gesell (1946) y Bayley (2006) como importante en el desarrollo de la locomoción bípeda.

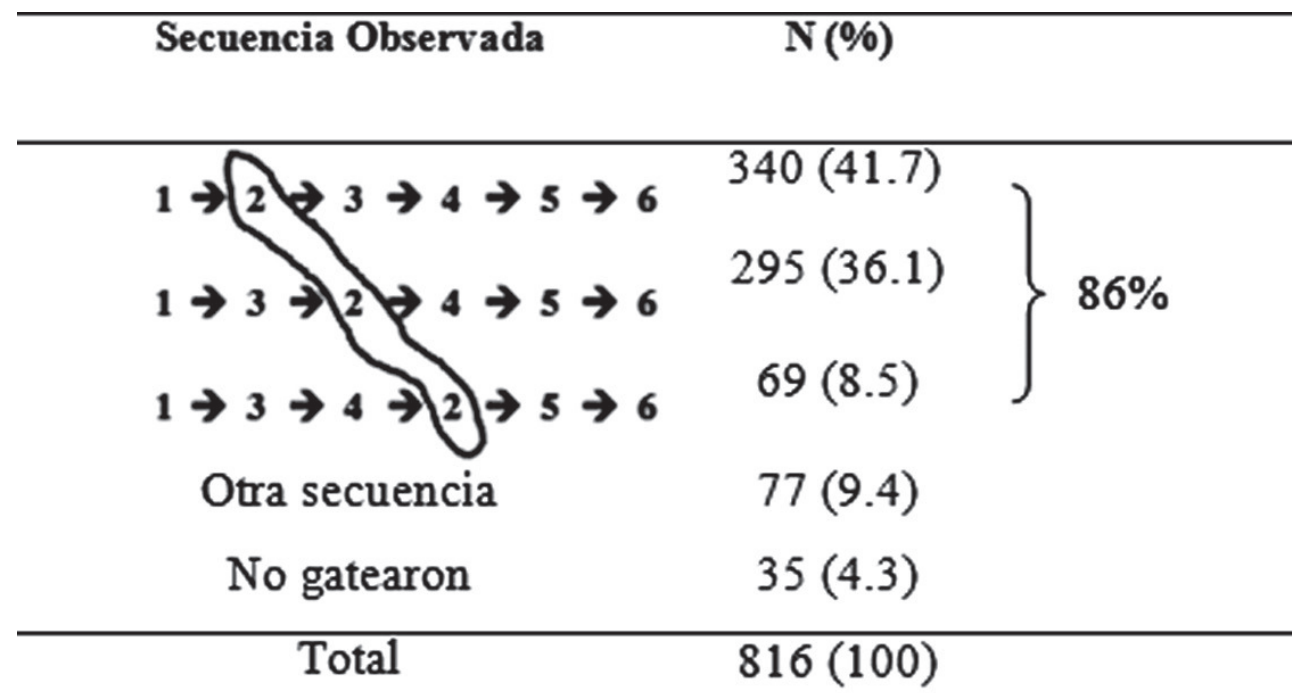

\begin{tabular}{lllll}
\hline Hitos: & l= & Sentarse sin ayuda & 2= & Arrastrarse con las manos y rodillas \\
& 3= & Pararse con ayuda & $\mathbf{4 =}$ & Caminar con ayuda \\
& 5= & Pararse por sí solo & 6= & Caminar por sí solo
\end{tabular}

Figura 1. Secuencias observadas en la adquisición de los seis hitos motores gruesos en el estudio normativo OMSDM (Adaptado de WHO Multicentre Growth Reference Study Group, 2006b)

Esta observación podría justificar la exclusión del gateo para posteriores consideraciones en este informe ya que la intención es centrarse en los hitos que siguen un orden de desarrollo. Sin embargo, hay que tener en cuenta que la exclusión no debe interpretarse como una atribución de menor valor en el desarrollo del gateo en comparación con los otros hitos motores en general, o de otros hitos relacionados con la locomoción bípeda, en particular (Adolph, Vereijken \& Denny, 1998; Campos \& Bertenthal, 1990). La información refleja la preocupación 
por la posibilidad de que el gateo no contribuya significativamente a la formación del caminar sino que constituya una estructura subyacente de desarrollo. Aún así, el gatear es sin duda un precursor de la movilidad (Adolph, Berger \& Leo, 2011) que muestra una mayor variabilidad explicada por la diversidad cultural, a comparación de otros hitos de motricidad gruesa.

En relación al orden de emergencia y la edad de adquisición, la Figura 2 ilustra la adquisición ordenada de ocho hitos motores gruesos en muestras de bebés y niños de EE.UU. (Capute, Shapiro, Palmer, Hoss, \& Wachtel, 1965), Nepal (Siegel, et al., 2005), Indonesia (Pollitt, Husaini, Harahp, Halati, Nugrahni \& Sherlock, 1994), y Zanzíbar (Kariger, Stoltzfus, Olney, Sazawal, Black, Tielsch, Frongillo, Khalfan \& Pollitt, 2005). Las muestras seleccionadas de Asia y África son niños económicamente pobres y mal alimentados, mientras que las otras dos muestras representaron a sus respectivas poblaciones nacionales. La muestra de Gran Bretaña fue excluida del análisis debido a que incluye solo dos hitos.

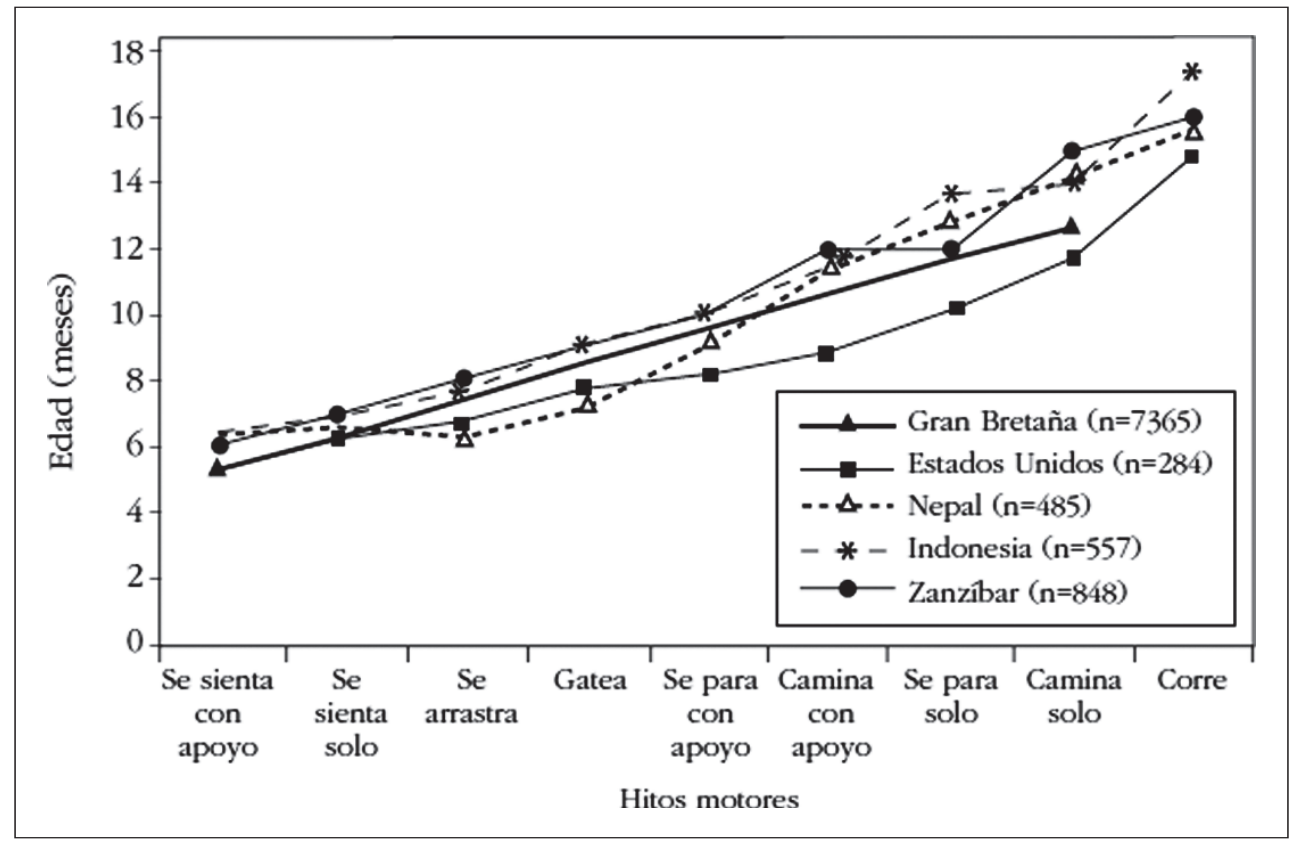

Figura 2. Ordenamiento y edad en la adquisición de nueve motores gruesos en nińos entre 3 y 18 meses de vida, expuestos a diferentes condiciones culturales y socioeconómicas (Adaptado de Pollitt, León \& Cueto, 2007) 
La muestra de Estados Unidos (Capute, et al., 1965) es representativa de los niños entre los 5 y 24 meses de edad en ese país, con tasas de desnutrición, anemia ferropénica y enfermedades contagiosas que no constituyen un problema grave de salud pública. Así, se evidencia que, entre los 2 y los 4 años de edad, hay un $2 \%$ de prevalencia de anemia ferropénica, mientras que $6,4 \%$ de los menores a los 5 años de edad tenían una talla por debajo del quinto percentil de la distribución respectiva (HANES 2000, en Center for Disease Control, 2002).

El estudio de Nepal (Siegel, Stoltzfus, Kariger, Katz, Khatry, Leclerq, Pollitt \& Tielsch, 2005), examinó el efecto de los factores nutricionales en la adquisición del caminar en una cohorte transversal de 485 niños de 4 a 17 meses de edad agrupados de acuerdo a su edad, sexo, raza, y nivel socioeconómico. En la muestra, el 58\% de los nińos padecían anemia (hemoglobina $<105 \mathrm{~g} / \mathrm{L}$ ), 2,1\% eran considerados como gravemente anémicos (hemoglobina $<70 \mathrm{~g} / \mathrm{L}$ ), mientras el $43 \%$ presentaban anemia por deficiencia de hierro (hemoglobina $<105 \mathrm{~g} / \mathrm{L}$; $\mathrm{EP} \geq 90 \mu \mathrm{mol} / \mathrm{mol})$. El 33,7\% de los niños evidencian un retraso en el crecimiento. Modelos de análisis multivariado que controlaron la edad, sexo, raza, y nivel socioeconómico pusieron de manifiesto que los niños con una mayor talla para la edad, mayor peso para la talla, ausencia de anemia, y consumo de carne, caminaron a una edad más temprana que los nińos con retraso en el crecimiento, anemia, y falta de consumo de carne. Se concluye que el crecimiento, la anemia, y la dieta se encuentran independientemente asociados a retrasos en el inicio de la locomoción bípeda entre los niños de corta edad.

El estudio de Indonesia (Pollitt et al., 1994) se centró en la asociación entre el crecimiento físico y el desarrollo motor grueso, especialmente en la adquisición de la locomoción, en 557 niños de 3 a 18 meses de edad de una población seleccionada de siete plantaciones de té en Java Occidental. El desarrollo motor grueso fue evaluado mediante nueve hitos motores relacionados con la adquisición de la locomoción. En el caso de los niños y niñas, la edad estaba negativa y significativamente $(\mathrm{p}<, 01)$ correlacionada con la talla. En el subgrupo de menor edad (3 a 6 meses) la media del puntaje $z$ era $-0,81$ para los niños $y-0,79$ para las nińas, 
mientras que en el subgrupo de mayor edad (16 a 18 meses) las medias eran de $-2,51$ y $-1,95$ respectivamente. En ese breve periodo de tiempo, el retardo en el crecimiento cambió de leve a severo. Esto permite sugerir que la talla para la edad, pero no el peso para la talla, fue un predictor significativo del retraso o no, del desarrollo motor grueso. El efecto del peso para la edad en el desarrollo motor no fue estadísticamente significativo luego de considerar la talla para la edad.

Así mismo, el estudio evidencia una adquisición ordenada de ocho hitos motores gruesos (Figura 3), similar a lo reportado por WHO (2006b). Sin embargo, en Indonesia el gatear no mostró tanta variación como lo hizo en el estudio multicéntrico. La Figura 3 muestra la frecuencia acumulada de edad para cada hito. Así por ejemplo, a los ocho meses ninguno de los niños fue capaz de caminar sin ayuda, sin embargo, cerca del 90\% de ellos habían adquirido esta misma habilidad a los 17 meses.

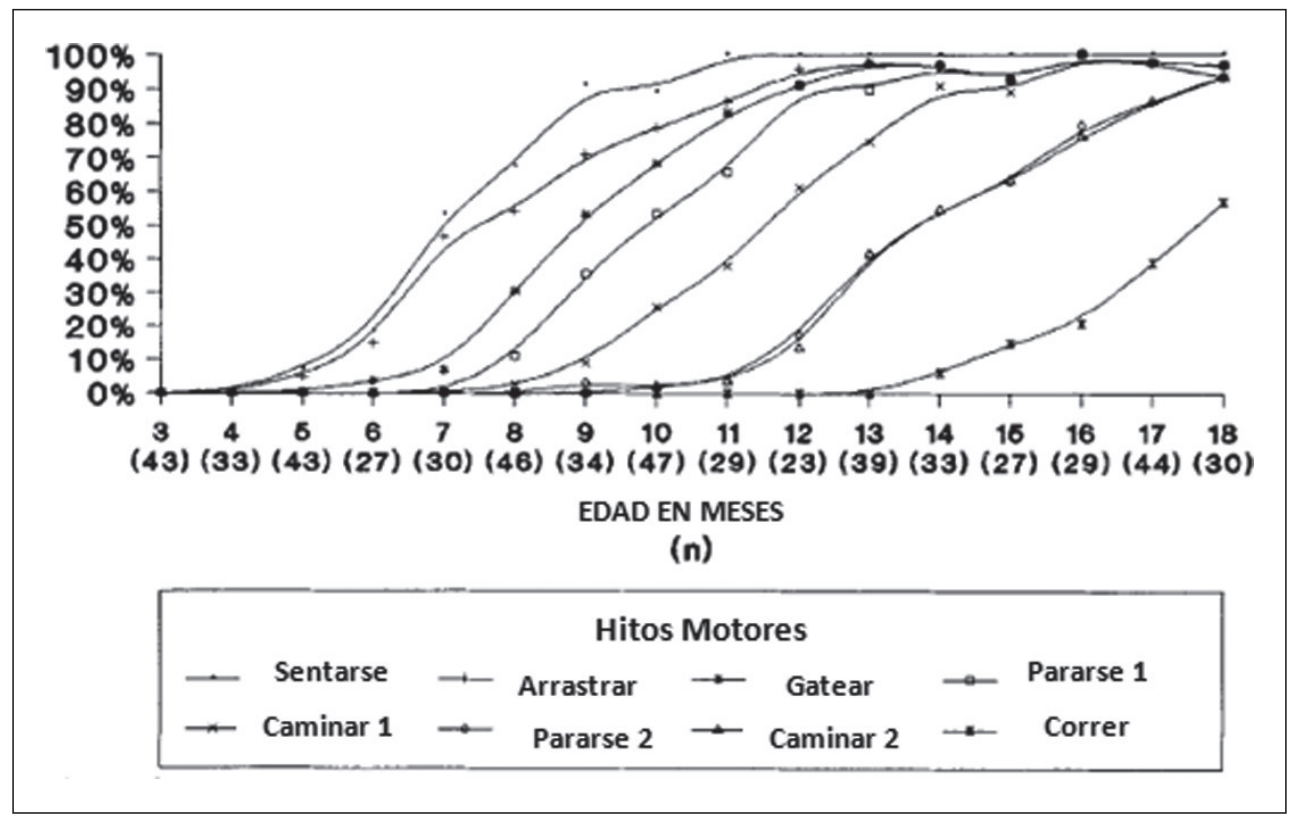

Figura 3. Secuencia de edad de adquisición de 8 hitos motores gruesos en el estudio de Indonesia (Adaptado de Pollitt et al., 1994)

En Zanzíbar (Kariger et al., 2005) se examinó la asociación entre la talla para la edad, peso para la talla y la anemia por deficiencia de hierro en el desarrollo de la locomoción en 646 niños de Zanzíbar. 
Los hitos del desarrollo motor grueso fueron evaluados utilizando una escala de 14 ítems, basada en la escala original de 17 ítems de McGraw (1943). Se trabajó con dos muestras. Una estaba conformada por 167 niños, de 6 a 18 meses de edad, que solo se arrastraban, pero no gateaban ni caminaban. Otra muestra incluía a 479 niños, de 9 a 18 meses de edad, que caminaban solos. De ambas muestras, el 82,6 y el 83,9\%, respectivamente, presentaban deficiencia de hierro y/o anemia (hemoglobina $<100 \mathrm{~g} / \mathrm{L}$, zinc protoporfirina $\geq 90 \mathrm{mmol} / \mathrm{mol}$ ). El $30,5 \% \mathrm{de}$ la muestra que se arrastraba y el $38,4 \%$ de la muestra que caminaba presentaban retraso en el crecimiento.

La curva de desarrollo para el logro de los hitos motores fue similar a la reportada en el estudio de Indonesia (Pollitt et al., 1994), evidenciándose además un retrasó en comparación con las normas de desarrollo de una evaluación estandarizada de las habilidades motrices básicas en infantes (Bayley, 1993). El estudio concluye que en una muestra de nińos mal alimentados, el crecimiento y el estado de la anemia por deficiencia de hierro son predictores significativos de la locomoción.

Las muestras de Indonesia, Nepal y Zanzíbar no eran representativas de las poblaciones respectivas, más bien fueron escogidas selectivamente entre poblaciones en las cuales la desnutrición, la anemia y las enfermedades contagiosas eran endémicas. La talla promedio de las muestras al segundo año de vida, en los tres estudios, estaba muy por debajo de los estándares de la Organización Mundial de la Salud. Como se observa al comparar las muestras, ni la alimentación ni la exposición continua a la pobreza modificaron la evolución ordenada del desarrollo motor grueso. El ordenamiento fue el siguiente; (1) sentarse sin apoyo; (2) pararse con apoyo; (3) gatear; (4) caminar con apoyo; (5) parase sin apoyo y (6) caminar sin apoyo.

Si bien existe una progresión ordenada de los hitos motores gruesos, el tiempo de adquisición de los hitos motores en la infancia y la niñez temprana, puede ser sensible a los efectos de la pobreza y la malnutrición. Para este análisis compararemos datos del estudio OMSDM con una investigación realizada en la isla de Pemba, que forma parte del archipiélago de Zanzíbar cerca a la costa de Tanzania (Kariger et al., 2005; Olney, Pollitt, Kariger, Khalfan, Ali, Tielsch, Sazawal, Black, Alen \& Stoltzfus, 2006). 
Mientras que los niños incluidos en el estudio OMSDM estaban sanos y bien nutridos, los infantes de la muestra de Zanzíbar, cuyas edades variaban entre los 5 y los 19 meses, provenían de áreas urbanas y rurales de uno de los cuatro distritos de la isla en donde las tasas de pobreza extrema, malaria, parasitosis intestinal, deficiencia de hierro y anemia y retardo en el crecimiento son muy elevadas en la población infantil bajo cualquier criterio internacional. En el estudio, se le daba individualmente a cada madre una serie de tarjetas que ilustraban la postura o el movimiento de un hito motor. La madre tenía que identificar los hitos que su infante ya había adquirido.

El histograma comparativo del desarrollo motor de los estudios respectivos que se observa en la Figura 4 es importante. La Figura 4 presenta, para cada una de las dos muestras, el percentil 50 de la distribución de la edad correspondiente para cada uno de los seis hitos. Es evidente que, independientemente de la progresión de los hitos motores que es similar en ambos estudios, los niños en Zanzíbar se retrasaron en comparación con los nińos que participaron en el estudio de la OMSDM (De Onis et al., 2004).

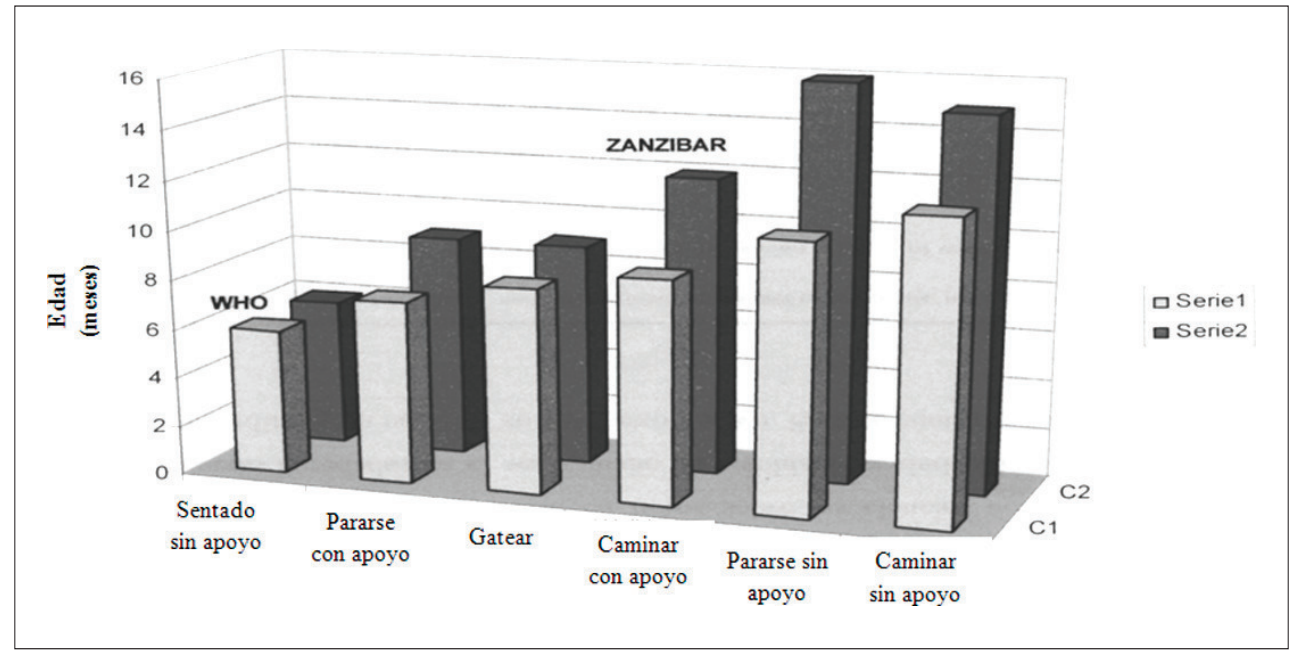

Figura 4. Percentil 50 de la edad de distribución correspondiente a cada uno de los 6 hitos motores gruesos en el estudio normativo OMSDM (De Onis et al., 2004) y el estudio longitudinal de Zanzíbar (Kariger et al., 2005). 


\section{Interpretaciones y comentarios}

Cualquier interpretación que se desee hacer de la diferencia en la información presentada debe tener en consideración los objetivos de los estudios y las características de los participantes. Los niños de la muestra de Estados Unidos y del estudio OMSDM presentaban mejores índices de salud y nutrición que los niños de las muestras de Indonesia, Nepal y Zanzíbar. Estas últimas muestras fueron escogidas de manera selectiva entre poblaciones con altos índices de desnutrición, anemia y enfermedades contagiosas. En el estudio de Pemba, por ejemplo, los participantes sufrían deficiencias de micronutrientes y malaria y la intención era evaluar los efectos sobre el desarrollo que podría traer una intervención nutricional que remediara las carencias nutricionales. Al contrario, el estudio de la OMS, al tener intenciones normativas, seleccionó como participantes a infantes sanos y bien nutridos.

La diferencia entre la historia de los participantes de los estudios de Pemba y la OMS, por ejemplo, explica porque la edad promedio de adquisición de un hito motor entre los infantes de Pemba era 2 a 3 mayor que la edad promedio en los infantes del estudio de la OMS. Es más, generalmente, la edad promedio en Pemba era similar al 99 percentil de los infantes de la OMS en el mismo hito. Cabe la posibilidad que esa diferencia en salud y nutrición también explique porque el gatear estaba nítidamente ordenado dentro de la secuencia de hitos en Pemba y no en la muestra internacional de la OMS.

Como se observa en las figuras 2, 3 y 4 no se evidencian diferencias entre las muestras comparadas en relación al orden en que se presentan los hitos motores. Sin embargo, se hace evidente que a partir de aproximadamente los 8 meses se presentan diferencias en la edad de adquisición de los hitos. Más aún, a los 8 meses de vida, las muestras de Indonesia, Nepal y Zanzíbar muestran un rezago en comparación con la muestra de Estados Unidos, como se observa en la figura 2. Esto sugiere que la estabilidad en el orden en que se presenta la emergencia de los hitos motores estudiados en todas las muestras se deba al papel de factores genéticos, mientras que variaciones en los factores 
socioculturales y de salud pueden haber contribuido de manera significativa en el origen de las diferencias entre las muestras en la edad de adquisición de los hitos motores.

Así, la exposición a diferentes factores de riesgo podría interferir la organización y desarrollo del sistema motor, alterando la mecánica de la locomoción. Estudios experimentales en roedores desnutridos muestran que un retraso en la maduración de los ganglios basales y el cerebelo contribuye al retraso del desarrollo motor (Thach, Goodkin \& Keating, 1992). Así mismo, la anemia ferropénica reduce la disponibilidad de hierro en regiones cerebrales asociados con la movilidad (Viteri \& Torun, 1981; Thomas, Grant \& Aubuchon-Endsley, 2009), contribuyendo también a una disminución de la oxigenación muscular (Celsing, Bloomstrand, Werner, Pihlstedt \& Ekblom, 1986) afectando la bioquímica y la biodinámica muscular (Paul, Dalley, Felt \& Beard, 2004). Por otro lado, estudios de campo en niños mal nutridos sugieren que un déficit crónico de la ingesta de energía produce retraso en el desarrollo motor así como actividad motora reducida (Chavez \& Martinez, 1982; Haas \& Brownlle, 2001; Pollitt \& Oh, 1994; Pollitt, Huang \& Jahari, 1999). Lo anterior traería como resultado que el niño expuesto a estas condiciones no caminará a la edad en que caminan los niños sanos y bien nutridos, afectando a su vez, otras áreas como el desarrollo mental (Adolph, 1997; Murray, Veijola, Moilanen, Miettunen, Glahn, Cannon, Jones \& Isohani, 2006; Roebers \& Kauer, 2009) y socioemocional (Bertenthal, Campos \& Barret, 1984; Biringen, Emde, Campos \& Appelbaum, 1995; Biringen, Emde, Campos \& Appelbaum, 2008).

La exposición del niño a un tratamiento nutricional adecuado puede equilibrar, en corto plazo, el rezago en el desarrollo motor (Jahari, SacoPollitt, Husaini \& Pollitt, 2000). Estudios experimentales ejecutados en Indonesia (Idrjadinata \& Pollitt, 1993; Jahari, et al., 2000), Zanzibar (Stoltzfus, Kvalsvig, Chwaya, Materosor, Albonico, Tielsch, Savioli \& Pollitt, 2001) y Costa Rica (Shafir, Angulo-Barroso, Calatroni, Jimenez \& Lozoff, 2006) muestran los beneficios del tratamiento de la anemia ferropénica y de suplementos diarios de energía y micronutrientes en el desarrollo motor de los nińos durante los primeros ańos de vida. 
Prácticas de crianza relacionadas con el desarrollo motor son consideradas también un factor subyacente importante (Adolph, 2010; Bril \& Breniere, 1989). Factores culturales que enriquecen e intensifican la estimulación de movimientos en los infantes aceleran el inicio del desarrollo de habilidades motoras gruesas. Por ejemplo, culturas africanas y caribeñas realizan diversas prácticas de cuidado de los niños que incluyen ejercicios formales que estimulan el desarrollo de habilidades motoras gruesas particulares (Hopkins, 1976; Hopkins \& Westra, 1988; Super, 1976).

Así mismo, factores contextuales que restringen y reducen la estimulación pueden retrasar la aparición de determinadas habilidades motrices (Adolph, 2010). Al norte de China (Mei, 1994) y en los andes peruanos (Panez, Silva \& Silva, 2007) los niños, durante los primeros 12 a 24 meses de vida, pasan muchas horas diarias al interior de pequeńos sacos llenos de arena fina, que reemplazan el empleo de pañales, o sobre la espalda de su madre sostenidos por una manta denominada llyclla, impidiendo al nińo mayores oportunidades para la práctica del caminar de manera autónoma. Como consecuencia, se limita sus posibilidades de desplazamiento mostrando ciertos retrasos para caminar en comparación con niños de la misma región que no presentan restricciones en sus movimientos.

Por otro lado, la interacción de un pequeño tamaño corporal, retraso en el desarrollo del motor y actividad motora limitada pueden inducir a padres y/o cuidadores a tratar a los niños como menores para su edad (Pollitt, 2000) imponiendo restricciones sobre el niño y limitando su independencia física para explorar el medio ambiente. Un estudio en una comunidad rural mexicana (Chávez \& Martínez, 1982) reportó que un suplemento alimenticio recibido por algunas madres y niños favoreció la relación entre los padres y sus hijos, en la medida que los niños incrementaron su velocidad de crecimiento físico, se movilizaban más fácilmente y se volvieron más activos.

Es importante también resaltar la influencia que puede tener el ambiente biofísico sobre el crecimiento y desarrollo del organismo en poblaciones que habitan zonas geográficas de gran altura (Monge 
\& León-Velarde, 2003; Virués-Ortega, Garrido, Javierre \& Kloezeman, 2006). El peso y la talla al nacer son consistentemente menores en poblaciones por encima de los 2500 y 3000 metros sobre el nivel del mar (Saco-Pollitt, 1981, 1989), independientemente del nivel socioeconómico debido probablemente a la hipoxia fetal durante el tercer trimestre del embarazo. Esto es importante si tenemos en cuenta que entre infantes sanos y bien nutridos aquellos con más peso tienden a comenzar a caminar a edades tempranas (Adolph, 1997). Lo contrario se observa entre infantes con menor peso, debido probablemente a que presentan menor fuerza muscular para caminar a la edad esperada (Siegel et al., 2005).

Lo anterior se enmarca dentro de un modelo de sistemas dinámico que excluye la predeterminación de las conductas o hitos motores (Smith \& Thelen, 2003). La figura 5 muestra el modelo propuesto de la dinámica de adquisición de la locomoción bípeda y la influencia de ésta en las áreas de desarrollo mental y socioemocional a la luz de la teoría contemporánea del desarrollo psicobiológico (Gottlieb, Wahlste \& Lickliter, 2006; Gottlieb, 2007) que reconoce el desarrollo como un proceso multidimensional caracterizado por la emergencia de nuevas estructuras y funciones debido a la continua interacción y efectos recíprocos entre las dimensiones que componen los dominios en el ser humano y su entorno.

Una propuesta clave en el modelo es que además de los cambios cerebrales que intervienen en el desarrollo de la locomoción bípeda, éste depende en parte también de dos procesos relacionados entre sí. El primero hace referencia a las relaciones entre el crecimiento físico y el desarrollo motor en el niño. Por ejemplo, la adquisición de la locomoción depende en parte del desarrollo neuronal y en lo físico, del sistema biodinámico, permitiendo al niño mantener su equilibrio corporal y dar pasos hacia adelante. El otro proceso está referido a las interacciones e intercambios entre el nińo y su entorno social y físico, que dependen en parte del desarrollo del primero de los procesos. Por ejemplo, la comunicación verbal y no verbal con las personas o la observación de objetos y el espacio físico forman interacciones significativas de desarrollo. 
Los dos procesos descritos, se consideran parte integrante del desarrollo que determinan la dirección y las probabilidades de desviación de una trayectoria de desarrollo esperado (Pollitt, 2000).

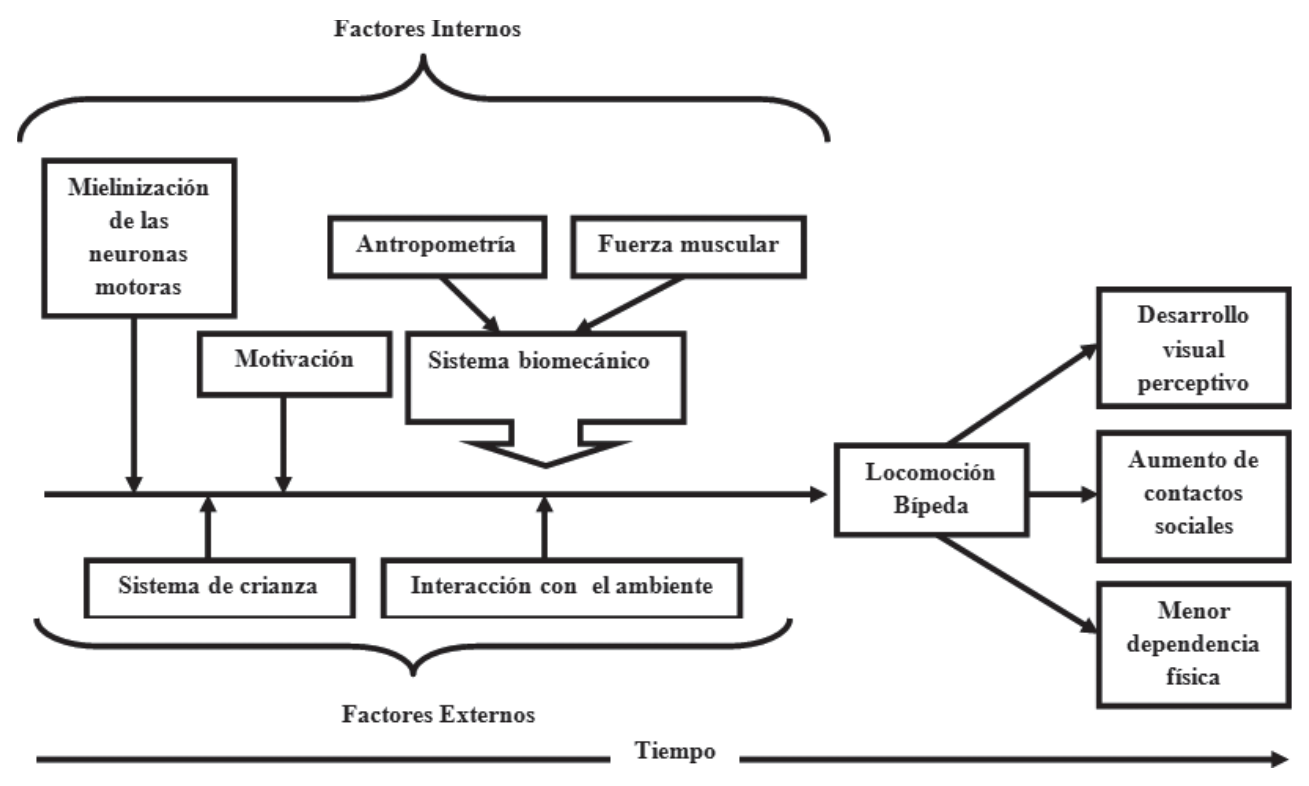

Figura 5. Dinámica de la adquisición de locomoción bípeda e influencia de ésta en otros aspectos del desarrollo mental y socioemocional.

El modelo evoca la complejidad del desarrollo de la locomoción debido a dos características inherentes. Primero, se basa en el concepto de cambio o transformación en el tiempo dentro y entre los dominios del modelo que llevan a la aparición de una nueva habilidad motora que se relaciona y contribuye, directa e indirectamente, a otras áreas de desarrollo así como a su medio físico y social. Desde una perspectiva ecológica (Bronfenbrenner \& Morris, 2006), el modelo permite describir que la temporalidad en la emergencia de cada nueva conducta motora se encuentra íntimamente relacionada con los procesos internos del organismo y la interacción entre éste y su entorno (Smith \& Thelen, 2003); conformando un conjunto de factores disponibles que contribuyen, dentro de un periodo determinado, hacia la formación del hito correspondiente, en donde los eventos previos determinan en parte los eventos presentes y futuros, y los eventos 
presentes se convierten en los eventos previos que afectan los eventos subsiguientes (Michel \& Tyler, 2005). Segundo, el modelo se refiere a los procesos que pueden conducir a una desviación gradual de un patrón de referencia que podría ser un grupo de control o de un criterio estadístico (Pollitt, 2000).

En conclusión, la evidencia sugiere la dificultad de generar datos normativos universales aplicables al desarrollo motor. Aunque el establecimiento de normas es útil para realizar generalizaciones acerca del tiempo de aparición de ciertas conductas y habilidades, estas deben de interpretarse con sumo cuidado, ya que las normas no evidencian las diferencias individuales de un niño a otro niño, en diferentes contextos de desarrollo, en el tiempo de logro de diversos hitos motores.

La variación físico-ecológica y la variación social de las poblaciones contribuyen a la variación en la configuración, ordenamiento y edad en la que emergen los hitos motores (Chisholm, 1978; Devine, 1985; Hadders-Algra, 2002; Tan, Karaca, Tan, Yilmlaz, Bagci, Ozcur \& Pence, 2008; Thelen, Scott \& Fogel, 1987). En el estudio de la OMSDM, si bien las normas buscaban representar el crecimiento normal independientemente de la etnia, nivel socioeconómico y tipo de alimentación, el emplear muestras de infantes con ausencia de problemas de salud, privaciones ambientales, económicas y que hayan nacido en ciudades ubicadas a una altitud por debajo de los $1500 \mathrm{msnm}$ (de Onis et al, 2004), traería como consecuencia que los resultados solo serían aplicables a una población similar a la "población de referencia" y no a otras con altos índices de desnutrición, infecciones y ubicadas a más de 1500 metros sobre el nivel del mar. La ausencia de variables nutricionales, culturales y biofísicas en la definición de normas del desarrollo motor traería como consecuencia, una inadecuada identificación de niños con retraso en ciertas habilidades motoras o que tiene un desarrollo normal, aunque en realidad, no sobrepase el percentil seleccionado para la población de referencia (Ponce de León, 2008). 


\section{Referencias}

Adolph, K. (1997). Learning in the development of infant locomotion. Monographs of the Society for Research in Child Development, 62, $1-140$.

Adolph, K., Vereijken, B. \& Denny, M. (1998). Learning to crawl. Child Development, 69(5), 1299-1312.

Adolph, K. (2010). Motor skill. En M. Bornstein (Ed.), Handbook of cultural developmental science (pp. 61-88). Nueva York: Psychology Press.

Adolph, A.; Berger, S. \& Leo, A. (2011). Developmental continuity? Crawling, cruising, and walking. Developmental Science 14(2), 306-318

Bertenthal, B., Campos, J. \& Barret, K. (1984). Self-produced locomotion: An organizer of emotional, cognitive, and social development in infancy. En R. Emde R. \& R. Harmon, Continuities and discontinuities in development. (pp. 175-210) Nueva York: Plenum.

Bayley, N. (1993). Bayley scales of infant development (2nd ed.). Nueva York: The Psychological Corporation.

Bayley, N. (2006). Bayley scales of infant and toddler development (3rd ed.). San Antonio: The Psychological Corporation

Biringen, Z., Emde, R., Campos, J. \& Appelbaum, M. (1995). Affective reorganization in the infant, the mother and the dyad-the role of upright locomotion and its timing. Child Development, 66, 499-514.

Biringen, Z., Emde, R., Campos, J. \& Appelbaum, A. (2008). Development of autonomy: Role of walking onset and its timing. Perceptual and Motor Skills, 106(2), 395-414.

Bril, B. \& Breniere, Y. (1989). Steady-state velocity and temporal structure of gait during the first six months of autonomous walking. Journal of Human Movement Science, 8, 99-122. 
Bronfenbrenner, U. \& Morris, P. (2006). The biological model of human development. En W. Damon \& R. Lerner (Eds.). Handbook of child psychology Vol. 1 Theoretical models of human development. 6ta. edición (pp. 793-828). Nueva Jersey: John Wiley y Sons, Inc.

Campos, J. J. \& Bertenthal, B. I. (1990). Locomotion and psychological development in infancy. En F. Morrison, K. Lord, \& D. Keating (Eds.), Applied developmental psychology: Psychological development in infancy (pp. 229-258). Nueva York: Academic Press.

Capute, A., Shapiro, B., Palmer, H., Hoss, A. \& Wachtel, R. (1965). Normal gross motor development: the influence of race, sex and socioeconomic status, Developmental Medicine and Child Neurology, 27, 635-645.

Celsing, F., Bloomstrand, B., Werner, B., Pihlstedt, P. \& Ekblom, B. (1986). Effects of iron deficiency on endurance and muscle enzyme activity in man. Medical Science and sports exercise, 18, 156-161.

Center for Disease Control (2002). Iron deficiency anemia, MMWR, 51, 897-899.

Chávez, A. \& Martínez, C. (1982). Growing up in a developing community. Cambridge, MA: Universidad de las Naciones Unidas.

Chisholm, J. (1978). Swaddling, cradleboards and development of children. Early Human Development 2(3), 255-275.

De Onis, M., Garza, C., Victora, C., Onyango, A., Frongillo, E., Martines, J. (2004). WHO Multicentre Growth Reference Study: planning, study design, and methodology. Food Nutrition Bulletin, 25(1) (supl. 1), S15-S26.

De Onis, M., Garza, C., Onyango, A. \& Martorell, R. (2006). WHO Child Growth Standards. Acta Pediatrica, 95(supplement 450), 5-101.

Devine, J. (1985). The versatility of human locomotion. American Anthropologist, 87(3), 550-570. 
Feldman, R. (2007). Desarrollo psicológico a través de la vida. México D. F.: Pearson Educación.

Gesell, A. (1946). The ontogénesis of infant behavior. En L. Carmichael, (Ed.), Manual of child psychology (pp. 295-331). Nueva York; John Wiley.

Gottlieb, G., Wahlsten, D. \& Lickliter, R. (2006). The significance of biology for human development. A developmental psychobiological systems view. En W. Damon \& R. Lerner (Eds.), Handbook of child psychology Vol. 1 Theoretical models of human development. 6ta edición (pp. 210-257). Nueva Jersey: John Wiley y Sons, Inc.

Gottlieb, G. (2007). Probabilistic epigenesis. Developmental Science, 10(1), 1-11.

Haas, J. \& Brownlle, T. (2001). Iron deficiency and reduced work capacity: a critical review of the research to determine a causal relationship. Journal of Nutrition, 131, 676S-690S.

Haas, J., Baker, P. \& Hunt, E. (1977). The effects of high altitude on body size and composition of the newborn infant in Southern Peru. Human Biology, 49(4), 611-628.

Hadders-Algra, M. (2002). Variability in infant motor behavior: A Hallmark of the Healthy nervous system. Infant Behavior \& Development 25(4), 433-451.

Hopkins, B. (1976). Culturally determined patterns of handling the human infant. Journal of Human Movement Studies, 2, 1-27.

Hopkins, B. \& Westra, T. (1988). Maternal handling and motor development. An intracultural study. Genetic, Social and General Psychology Monographs, 114, 379-408.

Huicho, L. \& Pawson, G. (2003). Crecimiento y desarrollo. En C. Monge \& F. León-Velarde (Eds.), El reto fisiológico de vivir en los Andes. Lima: Instituto Francés de Estudios Andinos.

Idrjadinata, P. \& Pollitt, E. (1993). Reversal of development delays in infants treated with iron. Lancet, 341, 1-4. 
Jahari, A., Saco-Pollitt, C., Husaini, M. \& Pollitt, E. (2000). Effects of an energy and micronutrient supplement on motor development and motor activity in undernourished children in Indonesia. European Journal of Clinical Nutrition, 54, S60-S68. Kariger, P., Stoltzfus R., Olney, D., Sazawal S., Black, R., Tielsch, J., Frongillo, E., Khalfan, S. \& Pollitt E. (2005), Iron deficiency and physical growth predict attainment of walking but not crawling in poorly nourished Zanzibar infants. Journal of Nutrition, 135, 814-819.

Kilbride, P. (1980). Sensorimotor behavior of Baganda and Samia infants. Journal of Cross-Cultural Psychology, 11, 131-152.

McGraw, M. (1943). The neuromuscular maturation of the human infant. Nueva York: Columbia University Press.

Mei, J. (1994). The northern Chinese custom of reading babies in sandbags. Implications for motor and intellectual development. En H. van Rossum \& J. Laszlo (Eds.), Motor development: Aspects of normal and delayed development. Amsterdam: VU Uitgeverij.

Michel, G. \& Tyler, A. (2005). Critical period: A history of the transition from questions of when, to what, to how. Developmental Psychobiology 46(3), 156-162.

Monge, C. \& León-Velarde, S. (2003). El reto fisiológico de vivir en los Andes. Lima: Instituto Francés de Estudios Andinos.

Murray, G., Veijola, J., Moilanen, K., Miettunen, J., Glahn, D., Cannon, T., Jones, P. \& Isohani, M. (2006). Infant motor development is associated with adult cognitive categorisation in a longitudinal birth cohort study. Journal of Child Psychology and Psychiatry, 47(1), 25-29.

Olney, D., Pollitt, E., Kariger, P., Khalfan, S., Ali, N., Tielsch, J., Sazawal, S., Black, R., Allen, L. \& Stoltzfus, R. (2006). Combined iron and folic acid supplementation with and without zinc reduces time to walking unassisted among Zanzibar infants 5-11 months old. Journal of Nutrition, 136, 2427-2434. 
Panez, R., Silva, G. \& Silva, M. (2007). El emprendimiento infantil en los Andes. Un modelo de promoción de capacidades para niños de o a 3 años. Lima: Panez \& Silva Consultores.

Paul, T., Dalley, C., Felt, B. \& Beard, J. (2004). Neonatal behavior and development are affected by early iron deficiency, FASEB Journal, 18, 49.

Pawson I., Huicho L., Muro M., \& Pacheco A. (2001). Growth of children in two economically diverse Peruvian high-altitude communities. American Journal Human Biology, 13, 323-340.

Pollitt, E. \& Oh, S.-Y. (1994). Early supplementary feeding, child development, and health policy. Food and Nutrition Bulletin, 15(3), 208-214.

Pollitt, E., Husaini, M., Harahp H., Halati, S., Nugrahni, A. \& Sherlock, A. (1994). Stunting and delayed motor development in West Java. American Journal of Human Biology, 6, 627-635.

Pollitt, E., Huang, J. \& Jahari, A. (1999). A developmental function off motor activity among nutritionally at-risk children. Food and Nutrition Bulletin, 20(1), 100-107.

Pollitt, E. (2000). A Development view of the undernourished child: background and purpose of the study in Pangalengan, Indonesia. European Journal of Clinical Nutrition, 54(2), S2-S10.

Pollitt, E., León, J. \& Cueto, S. (2007). Desarrollo infantil y rendimiento escolar. En Grupo de Análisis para el Desarrollo (Ed.), Investigación, Politicas y Desarrollo en el Perú (pp. 486-535). Lima: Grupo de Análisis para el Desarrollo.

Pollitt, E. \& Caycho, T. (2010). El desarrollo motor como indicador del desarrollo infantil durante los primeros dos años de vida. Revista de Psicología-Pontificia Universidad Católica del Perú, 28(2), 385-413.

Ponce de León, S. (2008). The WHO multicentre growth reference study and altitude above sea level. An Example of Hypsometric bias? High Altitude Medicine \& Biology, 9(3), 249-251. 
Robson, P. (1984). Prewalking locomotor movements and their use in predicting standing and walking. Child: Care, Health, and Development, 10, 317-330.

Roebers, C. \& Kauer, M. (2009). Motor and cognitive control in a normative simple. Development Science, 12(1), 175-181.

Saco-Pollitt, C. (1981). Birth in the Peruvian Andes: physical and behavioural consequences in the neonate. Child Development, $52,839-846$.

Saco-Pollitt, C. (1989). Ecocultural context and developmental risk: birth in the high altitudes (Peru). En K. Nugent, B. Lester \& T. Brazelton (Eds.), The cultural context of infancy: Biology, culture, and infant development (pp. 3-25). Westport, CT: Ablex Publishing.

Shafir, T., Angulo-Barroso, R., Calatroni, A., Jimenez, E. \& Lozoff, B. (2006). Effects of iron deficiency in infancy on patterns of motor development over time. Human Movement Science, 25, 821-838.

Siegel, H., Stoltzfus, R., Kariger, P., Katz, J., Khatry, S., Leclerq, S., Pollitt, E. \& Tielsch, J. (2005). Growth indices, anemia, and diet independently predict motor milestone acquisition of infants in South Central Nepal. Journal of Nutrition, 135(12), 2840-2844.

Smith L, \& Thelen, E. (2003). Development as a dynamic system. Trends in cognitive science, 7, 343-348.

Stoltzfus, R., Kvalsvig, J., Chwaya, H., Materosor, A., Albonico, M., Tielsch, J., Savioli, L. \& Pollitt, E. (2001). Effects of iron supplementation and anthelminthic treatment on motor and language development of Zanzibari preschool children. Londres: British Medical Journal Press.

Super, C. (1976). Environmental effects on motor development. The case of African infant precocity. Developmental Medicine and Child Neurology, 18, 561-567. 
Tan, Ü., Karaca, S., Tan, M., Yilmaz, B., Bagci, N., Ozkur, A. \& Pence, S. (2008). Unertan synfrome: A case series demonstrating human devolution. International Journal of Neuroscience 118(1), 1-25

Thach, W., Goodkin, H. \& Keating, J. (1992). The cerebellum and the adaptive coordination of movement. Annual Review of Neuroscience, 15, 403-442.

Thelen, E., Scott Kelso, J. \& Fogel, A. (1987). Self-Organizing systems and infant motor development. Developmental Review, 7(1): 39-65.

Thomas, D., Grant, S., Aubuchon-Endsley, N. (2009). The role of iron in neurocognitive development. Developmental Neuropsychology, 34(2), 196-222.

Virués-Ortega, J., Garrido, E., Javierre, C. \& Kloezeman, K. (2006). Human behavior and development under high-altitude conditions. Developmental Science, 9 (4), 400-410

Viteri. F. \& Torun, B. (1981). Nutrition, physical activity and growth. En M. Ritzen, A. Peria, K. Hall, A. Larsson, A. Zetterberg $\&$ R. Zettersrom (Eds.), The biology of normal human growth (pp. 265-273). Nueva York: Raven Press.

WHO Multicentre Growth Reference Study Group (2006a). WHO Motor Development Study: Windows of achievement for six gross motor development milestones. Acta Paediatrica, 95(Suppl. 450), 86-95.

WHO Multicentre Growth Reference Study Group (2006b). Reliability of motor development data in the WHO Multicentre Growth Reference Study. Acta Paediatrica, 95(Suppl. 450), 47-55.

Wijnhoven, T., De Onis, M., Onyango, A., Wang, T., Bjoerneboe, G., Bhandari, N., Lartey, A. \& Al Rashidi, B. (2004). Assessment of gross motor development in the WHO Multicentre Growth Reference Study. Food Nutrition Bulletin, 25(1), S37-S45.

Recibido: 13 de mayo de 2012 Aceptado: 30 de octubre de 2012 